Revista do Departamento de Geografia
Universidade de São Paulo
www.revistas.usp.br/rdg

\title{
iSAM - Um sistema de indicadores para o monitoramento da Área de Proteção Ambiental Mananciais do Rio Paraíba do Sul
}

\author{
iSAM-Socioenvironmental indicators for monitoring the Mananciais do \\ Rio Paraíba do Sul Protected Area with Sustainable Use (APA-MRPS)
}

\author{
Sacha Maruã Ortiz Siani \\ Instituto Nacional de Pesquisas Espaciais \\ sacha@ortiz.com.br \\ Silvana Amaral \\ Instituto Nacional de Pesquisas Espaciais \\ silvana@dpi.inpe.br \\ Antônio Miguel Vieira Monteiro \\ Instituto Nacional de Pesquisas Espaciais \\ miguel@dpi.inpe.br
}

Recebido (Received): 25/08/2016 DOI: $10.11606 /$ rdg.v33i0.119663
Aceito (Accepted): 13/02/2017
Resumo: A Área de Proteção Ambiental Mananciais do Rio Paraíba do Sul (APA-MRPS) é um importante instrumento de planejamento territorial ambiental que tem como objetivo proteger os mananciais que abastecem mais de 15 milhões de pessoas no sudeste do Brasil. Este espaço protegido inclui áreas rurais e urbanas, com diferentes contextos sociodemográficos e níveis de conservação. $\mathrm{O}$ objetivo deste trabalho foi construir um sistema de indicadores espacialmente explícitos, que auxiliasse o diagnóstico socioambiental e o monitoramento da APAMRPS. O sistema, intitulado iSAM - Indicadores Socioambientais de Monitoramento da APA-MRPS, consiste em um conjunto de indicadores, construídos a partir de dados de sensoriamento remoto e dados censitários, que pretende identificar e localizar no espaço as condições de proteção dos mananciais e os diferenciais sociodemográficos da população residente. A unidade Jaguari foi selecionada para análise, de modo a demonstrar a capacidade do iSAM de representar os diferenciais socioterritoriais existentes nessa unidade de conservação. O iSAM é indicado para apoiar a gestão e o monitoramento sistemático da APA-MRPS. O arcabouço conceitual criado neste trabalho para lidar com a APA-MRPS, com adaptações, pode ser utilizado para a construção de outros sistemas de indicadores no contexto do monitoramento e planejamento territorial de outras áreas protegidas.

Palavras-chave: Unidade de Conservação; Diagnóstico socioambiental; Recursos hídricos; Desenvolvimento sustentável; Avaliação.

\begin{abstract}
The Mananciais do Rio Paraíba do Sul Protected Area with Sustainable Use (APA-MRPS) is an important environmental and territorial planning instrument that aims to protect the water sources that supply more than 15 million people in the southeast of Brazil. This protected area includes rural and urban areas, with different sociodemographic contexts and conservation levels. This study aims to develop a spatially explicit indicator system, which would assist a socioenvironmental diagnosis and monitoring APA-MRPS. The system, called iSAM Socioenvironmental Indicators for Monitoring APAMRPS, consists of a set of indicators, built from remote sensing and census data, which aims to identify and locate in space the conditions for water sources protection and sociodemographic differences within the resident population. Jaguari unit was selected for analysis, in order to demonstrate the iSAM's capability to represent the existing socioterritorial differentials in this protected area. It will be useful to support planning, management, and systematic monitoring of the APA-MRPS. In addition, the conceptual framework created to deal with the APAMRPS, with adjustments, can be used to build other indicators systems in the monitoring and territorial planning of other protected areas.
\end{abstract}

Keywords: Conservation unit; Socioenvironmental diagnosis; Water resources; Sustainable development; Assessment; Evaluation. 


\section{INTRODUÇÃO}

A proteção dos mananciais de abastecimento da Bacia Hidrográfica do Rio Paraíba do Sul (BHPS) é um tema de relevância local e regional, uma vez que esta bacia é responsável pelo o abastecimento de cerca de 15 milhões de pessoas nos estados do Rio de Janeiro e São Paulo (AGEVAP, 2014). Entre as estratégias para mitigar processos de degradação dos recursos hídricos da região, está a Área de Proteção Ambiental (APA) dos Mananciais do Rio Paraíba do Sul (APA MRPS), que é objeto deste estudo. A APA MRPS foi criada em 1982 com o intuito de proteger os mananciais de abastecimento da BHPS (BRASIL, 1982). Sua configuração espacial abrange parte dos estados de São Paulo, Rio de Janeiro e Minas Gerais.

As APAs são Unidades de Conservação (UCs) de uso sustentável, destinadas a proteger e conservar a qualidade ambiental e os sistemas naturais existentes, visando melhorar a qualidade de vida da população local e a proteção dos ecossistemas. As APAs são instituídas sem a necessidade de desapropriar áreas e podem ser constituídas por terras públicas ou privadas, podendo compreender áreas urbanas e rurais e suas atividades econômicas inerentes (BRASIL, 2000).

Entretanto, apenas a criação de áreas protegidas não é suficiente para que este instrumento cumpra seus objetivos. Para sua efetividade, é fundamental estabelecer um plano de manejo, que defina objetivos e metas claras, e programas de ações, bem como informações qualificadas, a partir do monitoramento e de avaliações periódicas e sistemáticas, observando em que medida seus objetivos estão sendo cumpridos e a quais custos, para o manejo adaptativo (BENSUSAN, 2006). Desde a criação da APA MRPS, seu manejo e sua gestão, não foram plenamente implementados conforme determina o Sistema Nacional de Unidades de Conservação (SNUC) (BRASIL, 2000).

Os métodos sistemáticos de monitoramento de UCs mais utilizados no Brasil são voltados para biodiversidade, recursos naturais ou para avaliação e monitoramento da efetividade de gestão com ênfase na avaliação do papel do estado no controle do SNUC. Essas ferramentas não consideram os objetivos das UCs associados à sustentabilidade e suas múltiplas dimensões, nem como a sociedade é afetada ou beneficiada pela criação e gestão das UCs (MARINELLI, 2011).

O objetivo deste trabalho é construir um sistema de indicadores que auxilie o monitoramento e avaliação da efetividade das ações de manejo da APA-MRPS na conservação dos recursos hídricos, considerando as interações e consequências com e para a população residente. Esse sistema, intitulado iSAM - Indicadores Socioambientais de Monitoramento da Área de Proteção Ambiental Mananciais do Rio Paraíba do Sul, pretende identificar e localizar no espaço as condições de conservação de recursos e de vida da população que se inserem nesta UC. Apoiando-se em dados e técnicas de sensoriamento remoto orbital, dados censitários, e Sistemas de Informações Geográficas (SIG), busca-se construir um conjunto de indicadores espacialmente explícitos que são base para um sistema de indicadores, multiescalar e multidimensional, que trata das especificidades e das condições da APA-MRPS, mas que podem ser adaptados para outras UCs.

\section{2. ÁREA DE ESTUDO}

A área de estudo deste trabalho (Figura 1) compreende a porção paulista da APA-MRPS, que se distribui pelos três Estados que compõe a BHPS - São Paulo, Rio de Janeiro e Minas Gerais -, e é gerida pelo Instituto Chico Mendes de Conservação da Biodiversidade (ICMBio). Como os limites geográficos das unidades da APA-MRPS são conhecidos apenas em São Paulo, este estudo restringe-se às 15 unidades paulistas da APAMRPS. São 292.409 ha, que estendem-se desde a região metropolitana de São Paulo até o limite com o estado do Rio de Janeiro, e do alto Serra da Mantiqueira, limite com Minas Gerais, até a Serra do Mar. As unidades situam-se em 24 municípios do Vale do Paraíba. Criada por meio do decreto federal no 87.561 de 13 de setembro de 1982, a APA-MRPS tem o objetivo específico de proteger os mananciais de abastecimento da BHPS (BRASIL, 1982). 


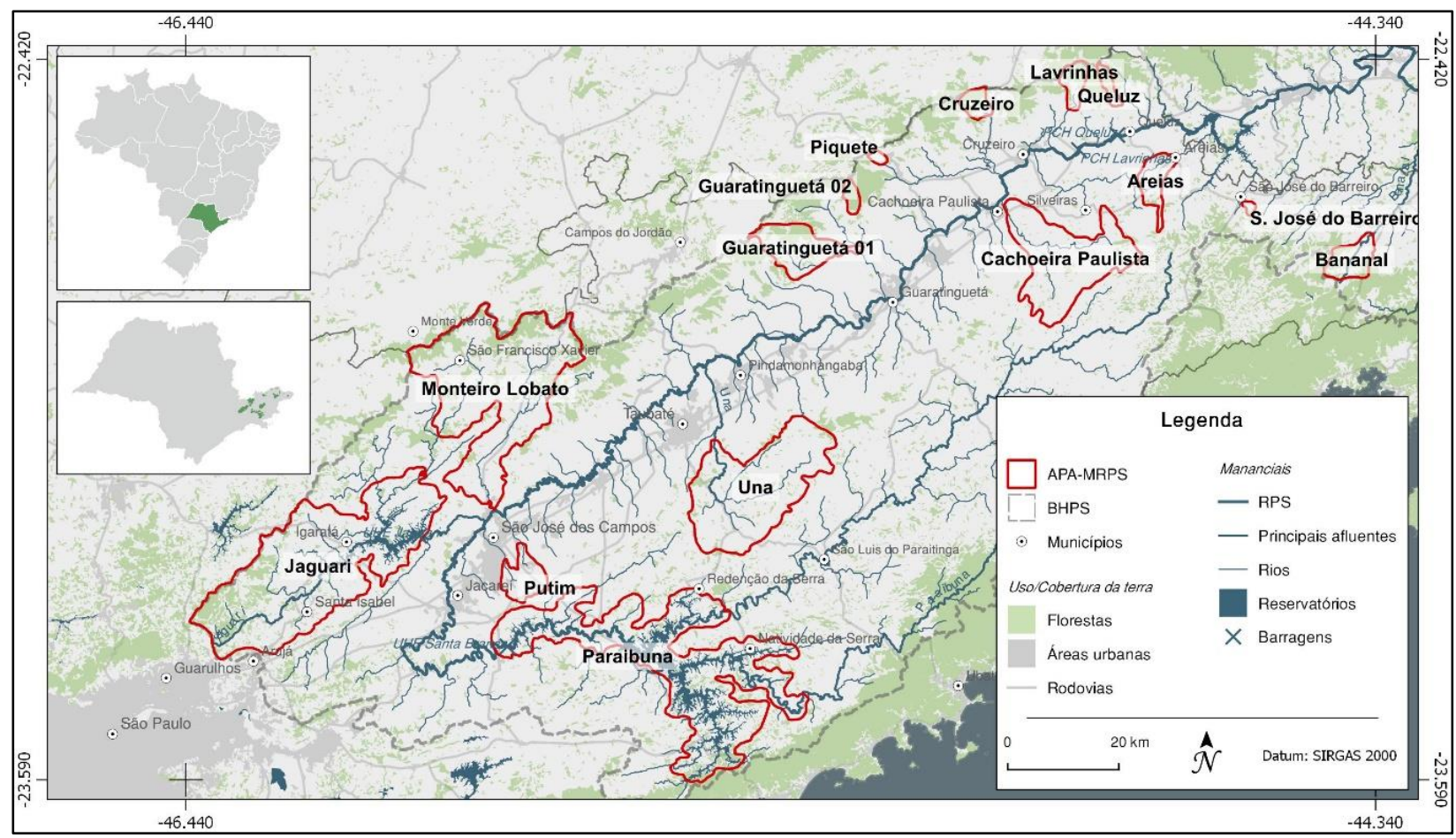

Figura 1: Área de estudo - unidades paulistas da APA-MRPS.

Fonte: Hidrografia: Agência Nacional de Águas (ANA); APA-MRPS: ICMBio; Limites estaduais: IBGE; Uso/cobertura da terra: SOSMA/INPE. Mapa organizado pelos autores.

\section{METODOLOGIA: A CONSTRUÇÃO DO SISTEMA DE INDICADORES}

\subsection{Modelo conceitual e definição das dimensões}

O conceito a ser operacionalizado neste trabalho tem como ponto de partida os objetivos gerais de UCs de uso sustentável, APAs, e os específicos da UC em estudo, a APA-MRPS.

As unidades de uso sustentável têm como objetivo básico compatibilizar a conservação da natureza com o uso sustentável de parcela dos seus recursos naturais (BRASIL, 2000). O SNUC entende como uso sustentável a "exploração do ambiente de maneira a garantir a perenidade dos recursos ambientais renováveis e dos processos ecológicos, mantendo a biodiversidade e os demais atributos ecológicos, de forma socialmente justa e economicamente viável" (BRASIL, 2000, Art. 2o). As APAs, por sua vez, têm como objetivos proteger a diversidade biológica, disciplinar o processo de ocupação e assegurar a sustentabilidade de uso dos recursos naturais. A APA-MRPS tem o objetivo particular de proteger os mananciais superficiais de abastecimento da BHPS (BRASIL, 1982; CEEIVAP, 1980). Por "proteção dos mananciais superficiais", entende-se o manejo dos recursos naturais, para manter ou melhorar as condições de fontes hídricas superficiais que possam ser utilizadas para atender às diversas demandas consuntivas (BRASIL, 2000; ANA, 2015).

Nota-se que a noção de sustentabilidade, ou desenvolvimento sustentável, foi institucionalizado pelo SNUC e está presente nos objetivos, principalmente, das UCs de uso sustentável, a fim de conciliar a conservação da biodiversidade e dos recursos naturais com a ocupação humana. Sendo assim, a definição do sistema de indicadores deste trabalho demanda uma abordagem multidimensional, baseada em uma tríade que represente a dimensão ecológica, a dimensão social e a dimensão econômica (SACHS, 2008).

Nesta abordagem, a dimensão ecológica diz respeito essencialmente aos processos e dinâmicas dos usos e coberturas da terra na APA MRPS. Estes processos associam-se diretamente à proteção da diversidade biológica, ao processo de ocupação, e à conservação dos recursos naturais, particularmente dos mananciais hídricos, para a manutenção da qualidade de vida e bem-estar das populações humanas. Esta dimensão parte do pressuposto que a composição e configuração da paisagem estão associadas à manutenção da biodiversidade e dos recursos hídricos.

Apesar de diversos fatores interagirem com a cobertura da terra para controlar os fluxos hidrológicos, apenas o uso e cobertura da terra podem ser modificados e manejados em escalas temporais compatíveis com as dinâmicas socioeconômicas, nas quais se estabelecem programas e políticas públicas para a conservação 
dos recursos hídricos. Sendo assim, variáveis como forma e relevo da área, apesar de importantes, não fazem parte do escopo deste sistema de indicadores.

A dimensão social identifica o perfil da população residente quanto às suas capacidades, conhecimentos e condições que lhes permitam, potencialmente dada as oportunidades, ampliar suas possibilidades econômicas e de qualidade de vida. Esta dimensão inclui os temas educação e saúde. A dimensãa econômica retrata a capacidade do domicílio como uma unidade produtiva, a estrutura de renda familiar e o acesso ao mercado de trabalho da população inserida na APA-MRPS. Deste modo, os indicadores incluídos nesta dimensão integram os temas trabalho, rendimento e seu padrão distributivo.

Dimensão Ecológica: Para representar a dinâmica de uso e cobertura nas unidades da APA-MPRS desde a sua criação e, assim, obter as estatísticas que constituem a dimensão Ecológica, utilizou-se o mapeamento de uso e cobertura da terra realizado por Siani (2016), que utilizou imagens ópticas da série Landsat para os anos de referência 1985, 1991, 2000, 2010 e 2015, para mapear as seguintes classes de uso ou cobertura da terra: 'Água', 'Área construída', 'Solo exposto', 'Floresta', 'Herbáceo/Arbórea', 'Pasto/Gramíneas', 'Mineração', 'Floresta monoespecífica', 'Rochas' e 'Não observado'.

Os indicadores da dimensão ecológica refletem a importância das classes de uso e cobertura da terra para a conservação dos recursos hídricos, traduzidos em métricas obtidas a partir do mapeamento de uso e cobertura da terra, calculadas para unidades de área regulares de $200 \times 200 \mathrm{~m}$. As métricas correspondem às proporções das classes mapeadas. Cada classe possui uma importância associada às suas relações com a proteção dos mananciais, conforme apresentado na Tabela 1.

Tabela 1: Indicadores representantes da dimensão Ecológica do iSAM.

\begin{tabular}{|c|c|}
\hline Indicador & Justificativa \\
\hline$\%$ Floresta & $\begin{array}{l}\text { Florestas têm maior potencial em fornecer serviços ecossistêmicos, e sua conservação implica na } \\
\text { proteção direta dos mananciais hídricos da bacia. Contribuem para a infiltração de água no solo; a } \\
\text { proteção do solo, evitando processos erosivos; proteção ripária; melhoria da qualidade da água; } \\
\text { regulação do fluxo de água; e produção de água (LIMA; FERRAZ; FERRAZ, 2013). A remoção da } \\
\text { floresta causa a fragmentação de habitats, a extinção de espécies, a interferência nos fluxos e processos } \\
\text { biológicos e de polinização, dentre outros danos à biodiversidade (NAGENDRA et al., 2013). }\end{array}$ \\
\hline $\begin{array}{c}\text { \%Floresta } \\
\text { monoespecífica }\end{array}$ & $\begin{array}{l}\text { Quando maduras, as 'Florestas monoespecíficas' contribuem para o processo de infiltração de água e } \\
\text { proteção do solo (LIMA, 2010). No corte raso ocorrem aumento na concentração de sedimentos em } \\
\text { suspensão nos mananciais, perdas de solo e nutrientes, prejudiciais à qualidade da água } \\
\text { (PENNINGTON; LAFFAN, 2004; STOTT et al., 2001). Florestas plantadas em áreas onde a floresta } \\
\text { natural foi removida podem ajudar a conservação da biodiversidade, fornecendo habitat complementar } \\
\text { e aumentando a conectividade entre fragmentos florestais (BROCKERHOFF et al., 2008). }\end{array}$ \\
\hline \%Herbáceo/ Arbórea & $\begin{array}{l}\text { Vegetação 'Herbáceo/Arbórea' por sua estrutura, copa, sub-bosque e serapilheira, tem menor potencial } \\
\text { que as florestas para cumprir funções ecológicas em benefício dos mananciais (LIMA; FERRAZ; } \\
\text { FERRAZ, 2013). Ainda que em menor grau, a classe 'Herbáceo/Arbórea' tem as mesmas vantagens } \\
\text { que a 'Floresta' em relação às classes com cobertura vegetal de menor porte ou não vegetada. }\end{array}$ \\
\hline \%Pasto/ Gramíneas & $\begin{array}{l}\text { A vegetação de gramíneas pouco contribui para proteção do solo e infiltração de água na terra. Na } \\
\text { pecuária, o pisoteio animal nas margens de corpos d'água podem causar compactação do solo e } \\
\text { desencadear processos erosivos. As atividades agrícolas fazem uso de substâncias químicas como } \\
\text { pesticidas, herbicidas, fertilizantes e resíduos animais, contaminantes dos mananciais (ALLAN, 2004). }\end{array}$ \\
\hline$\%$ Solo exposto & $\begin{array}{l}\text { Em regiões de 'Solo exposto', o solo é altamente impermeabilizado, favorecendo o escoamento } \\
\text { superficial, e contribuindo para a ocorrência/agravamento de processos erosivos e, consequentemente, } \\
\text { aumentar a produção de sedimentos que degradam os mananciais. }\end{array}$ \\
\hline \%Mineração & $\begin{array}{l}\text { Em áreas de 'Mineração', a água em contato com rochas mineralizadas pode ser contaminada por } \\
\text { metais pesados e representam uma fonte de alto potencial poluidor de águas subterrâneas e superficiais } \\
\text { (WENDLAND; MARIN, 2013). Há ainda, riscos inerentes à operação das minerações, como de } \\
\text { vazamento de rejeitos. }\end{array}$ \\
\hline \%Área construída & $\begin{array}{l}\text { Em áreas urbanas o solo é impermeabilizado com edificações e pavimentos, e a água é conduzida pelas } \\
\text { galerias de águas pluviais. Estas mudanças na cobertura reduzem a infiltração no solo, ocasionam } \\
\text { aumentos nas vazões médias dos corpos hídricos e produzem sedimentos, provocando assoreamento } \\
\text { dos corpos d'água e transporte de poluente agregado ao sedimento, o que contamina as águas fluviais } \\
\text { (TUCCI; MENDES, 2006). Os resíduos, sólidos e líquidos, produzidos em áreas urbanas são fontes } \\
\text { potenciais de poluição dos mananciais, caso sejam dispostos de maneira inadequada. }\end{array}$ \\
\hline
\end{tabular}

Fonte: Produzido pelos autores. 
A importância de cada classe foi traduzida em valores através da técnica de comparação pareada AHP (Processo Analítico Hierárquico), obedecendo a escala de valores proposta por Saaty (1977). A classe com maior importância foi 'Floresta' (40,3\%), seguida por 'Herbáceo/Arbórea' (23,9\%), 'Floresta monoespecífica' $(14,6 \%)$, 'Pasto/Gramíneas' (9,8\%), 'Solo exposto' (5,7\%), 'Área construída' (3,6\%) e 'Mineração' (2,0\%).

Dimensões Social e Econômica: Utilizou-se o conjunto de dados do universo dos Censos Demográficos de 2000 e 2010 (IBGE, 2010) para compor os indicadores das dimensões social e econômica, agrupados por setor censitário. A Tabela 2 descreve os indicadores selecionados para representar as dimensões Social e Econômica e suas respectivas justificativas.

Tabela 2: Indicadores representantes das dimensões Social e Econômica do iSAM.

\begin{tabular}{|c|c|}
\hline Indicador & Justificativa \\
\hline & Dimensão Social \\
\hline $\begin{array}{l}\text { \%Alfabetizados } \\
\quad(8-17 a)\end{array}$ & $\begin{array}{l}\text { A alfabetização, e outros conhecimentos básicos adquiridos a partir do ensino básico, fornecem } \\
\text { condições para que as pessoas tenham capacidade para processar informações, selecionando o que é } \\
\text { relevante, e continuar aprendendo. Ademais, ampliam as possibilidades futuras, desses jovens, de } \\
\text { inserção no mercado de trabalho (IBGE, 2015). }\end{array}$ \\
\hline $\begin{array}{l}\text { \%Responsáveis } \\
\text { alfabetizados }\end{array}$ & $\begin{array}{l}\text { A alfabetização entre os responsáveis amplia as possibilidades de acesso ao mercado de trabalho e } \\
\text { as possibilidades de incentivar seus filhos a frequentar a escola (IBGE, 2015). A educação da } \\
\text { população potencializa as chances de se implementar com sucesso as ações de manejo de uma UC } \\
\text { como a APA-MRPS; }\end{array}$ \\
\hline $\begin{array}{l}\text { \%Pessoas acima da } \\
\text { expectativa de vida (por } \\
\text { gênero) }\end{array}$ & $\begin{array}{l}\text { A existência de pessoas em idade superior à esperança de vida ao nascer sugere boas condições de } \\
\text { vida e de saúde da população em relação ao estado de maneira geral. Utilizou-se a expectativa média } \\
\text { do estado de São Paulo; }\end{array}$ \\
\hline $\begin{array}{l}\text { \%Cobertura de } \\
\text { abastecimento de água }\end{array}$ & $\begin{array}{l}\text { Acessibilidade à água de qualidade para consumo e em quantidade necessária para a higiene pessoal } \\
\text { interrompem os ciclos de muitas doenças que se proliferam pela água ou pela falta dela (HELLER, } \\
\text { 1998; UNITED NATIONS, 2007). Nesse sentido, trata-se de um indicador indireto das condições de } \\
\text { saúde da população; }\end{array}$ \\
\hline $\begin{array}{l}\text { \% Cobertur } \\
\text { coleta de } 1\end{array}$ & $\begin{array}{l}\text { A destinação adequada de resíduos sólidos constitui uma importante medida de prevenção de } \\
\text { doenças, posto que os resíduos dispostos incorretamente tendem a obstruir drenagens de águas } \\
\text { pluviais, causando enchentes e contaminando corpos hídricos, favorecendo a proliferação de doenças } \\
\text { de veiculação hídrica (IBGE, 2015). }\end{array}$ \\
\hline \multirow[t]{2}{*}{$\begin{array}{l}\% \text { Cobertura de } \\
\text { esgotamento sanitário }\end{array}$} & $\begin{array}{l}\text { O tratamento de esgotos é um dos mais importantes meios de prevenção de doenças (HELLER, } \\
\text { 1998). Além de terem influência na saúde e na qualidade de vida da população, os indicadores de } \\
\text { cobertura de esgotamento sanitário e de coleta de lixo expressam pressões sobre os recursos naturais, } \\
\text { especialmente os recursos hídricos, e nesse sentido, poderiam representar também a dimensão } \\
\text { ecológica do sistema de indicadores. }\end{array}$ \\
\hline & Dimensão Econômica \\
\hline $\begin{array}{l}\text { Rendimento médio do } \\
\text { responsável }\end{array}$ & $\begin{array}{l}\text { O rendimento dos responsáveis indica a capacidade de cobertura do orçamento doméstico, bem como } \\
\text { a capacidade de aquisição de bens e serviços; }\end{array}$ \\
\hline $\begin{array}{l}\text { \%Domicílios } \\
\text { próprios }\end{array}$ & $\begin{array}{l}\text { A presença de domicílios próprios indica maior estabilidade econômica da população. Por ser } \\
\text { correlacionado à renda familiar é uma alternativa de identificação do poder aquisitivo da população } \\
\text { (ANAZAWA, 2012; JANNUZZI, 2012); }\end{array}$ \\
\hline $\begin{array}{l}\text { Índice de Gini - renda } \\
\text { dos responsáveis }\end{array}$ & $\begin{array}{l}\text { O índice de Gini indica as desigualdades sociais oriundas da apropriação diferenciada do rendimento } \\
\text { pelos responsáveis (IBGE, 2015; JANNUZZI, 2012), complementando a análise do valor absoluto } \\
\text { do rendimento médio; }\end{array}$ \\
\hline $\begin{array}{l}\text { \%Responsáveis com } \\
\text { rendimento acima de um } \\
\text { sm (por gênero) }\end{array}$ & $\begin{array}{l}\text { Reflete a capacidade do sistema econômico em prover ocupação produtiva aos responsáveis por } \\
\text { domicílios que a desejam. O indicador desagregado por sexo evidencia desigualdades de gênero no } \\
\text { acesso ao mercado de trabalho (IBGE, 2015); }\end{array}$ \\
\hline $\begin{array}{c}\text { Razão de } \\
\text { dependência }\end{array}$ & $\begin{array}{l}\text { Mede a participação relativa do contingente populacional potencialmente inativo, que deveria ser } \\
\text { sustentado pela parcela da população potencialmente produtiva. Valores elevados indicam que a } \\
\text { população em idade produtiva sustentará uma grande proporção de dependentes, implicando em } \\
\text { encargos assistenciais para a sociedade (IBGE, 2015). }\end{array}$ \\
\hline
\end{tabular}

Fonte: Produzido pelos autores. 
A fim de verificar o grau de associação entre as estatísticas selecionadas, foram realizadas análises de correlação entre os indicadores simples, para a área de estudo, nos períodos analisados. Somente foram encontradas associações fortes $(>0,70)$ entre os indicadores \%Responsáveis com rendimento acima de 1 SM para homens e para mulheres. Ainda que correlacionados optou-se por mantê-los na análise para que fosse possível abordar as desigualdades de gênero.

Como os setores censitários podem ter pequenos volumes populacionais, para evitar a instabilidade em pequenas áreas (DRUCK et al., 2004), utilizou-se o estimador bayesiano empírico local (MARSHALL, 1991).

Para representar a heterogeneidade espacial dos setores censitários, como propostos por Amaral et al. (2012) e D’Antona, Bueno e Dagnino (2013), utilizou-se as informações da identificação de domicílios do Cadastro Nacional de Endereços para fins Estatísticos - CNEFE de 2010. No CNEFE, foram coletados os pares de coordenadas geográficas para as edificações em situação rural, e para edificações em situação urbana foram coletados apenas os endereços, que foram geocodificados utilizando o Google Maps Geocoding API.

Para integrar os dados de sensoriamento remoto e os dados censitários em uma única referência espacial, adotou-se a abordagem por espaço celular, ou seja, uma grade de células que permite compatibilizar informações de diferentes fontes e formatos integrando-os em uma mesma base espaço-temporal. Adotou-se células regulares com resolução de $200 \times 200 \mathrm{~m}$, o que corresponde a escala espacial mais refinada possível em função da capacidade de processamento disponível, e da restrição de que cada setor deveria conter pelo menos uma célula. Os atributos das células foram calculados em um SIG, a partir de dados armazenados nas camadas de mapeamento de uso e cobertura da terra, dos indicadores sociais e econômicos agregados por setores censitários e das edificações provenientes do CNEFE.

Por fim, foi aplicado o método dasimétrico binário, onde os indicadores socioeconômicos selecionados foram redistribuídos somente entre as células que continham uma ou mais edificações (pontos) provenientes do CNEFE.

\subsection{Modelo de cálculo e representação dos indicadores compostos}

Além dos indicadores simples descritos anteriormente, foi criado um indicador composto para cada dimensão do sistema de indicadores. Como os indicadores simples, sociais e econômicos têm diferentes unidades de medida, e diferentes intervalos de variação, eles foram escalonados em medidas adimensionais entre 0 e 1 , onde o valor máximo representa uma condição melhor.

Os indicadores compostos das dimensões Social e Econômica foram então calculados como a média aritmética dos indicadores simples normalizados, e novamente escalonados. Para a dimensão Ecológica, o indicador composto foi calculado como a média ponderada dos percentuais de classes de uso e cobertura da terra, tendo como fator de ponderação o resultado da AHP e, posteriormente, também passaram pela transformação de escala.

Os indicadores compostos foram representados por uma grade multidimensional, onde cada dimensão recebeu uma cor, similar à técnica para a composição colorida de imagens de sensoriamento remoto (JENSEN, 2007): as cores R (Vermelho), G (Verde), e B (Azul) representam as dimensões Econômica, Ecológica e Social, respectivamente Esta representação permitiu uma análise conjunta dos três indicadores compostos, referentes às dimensões do sistema, proporcionando capacidade de síntese, mas sem a perda de proximidade entre os conceitos e a medida que uma nova aglutinação dos indicadores traria.

\section{RESULTADOS E DISCUSSÕES}

O sistema de indicadores foi construído para todas as unidades da APA-MRPS, e apresentado em um painel de observações (ANAZAWA, 2012), que inclui o gráfico da dinâmica de uso e cobertura, os indicadores simples das dimensões Social e Econômica para os períodos 2000 e 2010 e os mapas dos indicadores compostos em composição RGB, também para os dois períodos.

A síntese dos diferentes contextos sociais, econômicos e ecológicos das unidades que compõem a APA MRPS são apresentados graficamente na Figura 2, considerando seus indicadores compostos das três dimensões do iSAM. 
Os resultados apontaram que, entre as unidades da APAMRPS, as que melhor cumprem a função ecológica de proteção dos mananciais são Guaratinguetá 2, Cruzeiro, Lavrinhas, Queluz, Piquete e Bananal, respectivamente. Estas unidades situam-se nas encostas das serras da Mantiqueira e da Bocaina, onde a topografia restringe a maioria dos usos da terra. Em relação às populações humanas inseridas na APA, a unidade do Putim se destaca por apresentar, em ambas as datas analisadas, as melhores condições sociais e econômicas, mas também as piores condições da paisagem para a proteção dos recursos hídricos. Em menores proporções em relação à unidade Putim, as unidades de Monteiro Lobato e Guaratinguetá 1 também apresentam boas condições sociais e econômicas. Observando a dinâmica entre 2000 e 2010: a) na dimensão Ecológica, Bananal, Paraibuna e Jaguari foram as que mais evoluíram, e Una, Cruzeiro e Putim as que mais pioraram; b) na dimensão Econômica, Cachoeira Paulista e Lavrinhas foram as únicas a melhorar, e as que mais pioraram foram Cruzeiro, Bananal e Jaguari; e c) na dimensão Social, por outro lado, apenas a unidade Cruzeiro retrocedeu, e as que mais evoluíram foram Bananal, Paraibuna, Cachoeira Paulista e Areias. Na seção a seguir, a análise detalhada da unidade Jaguari demonstra o potencial do painel de observações do iSAM.

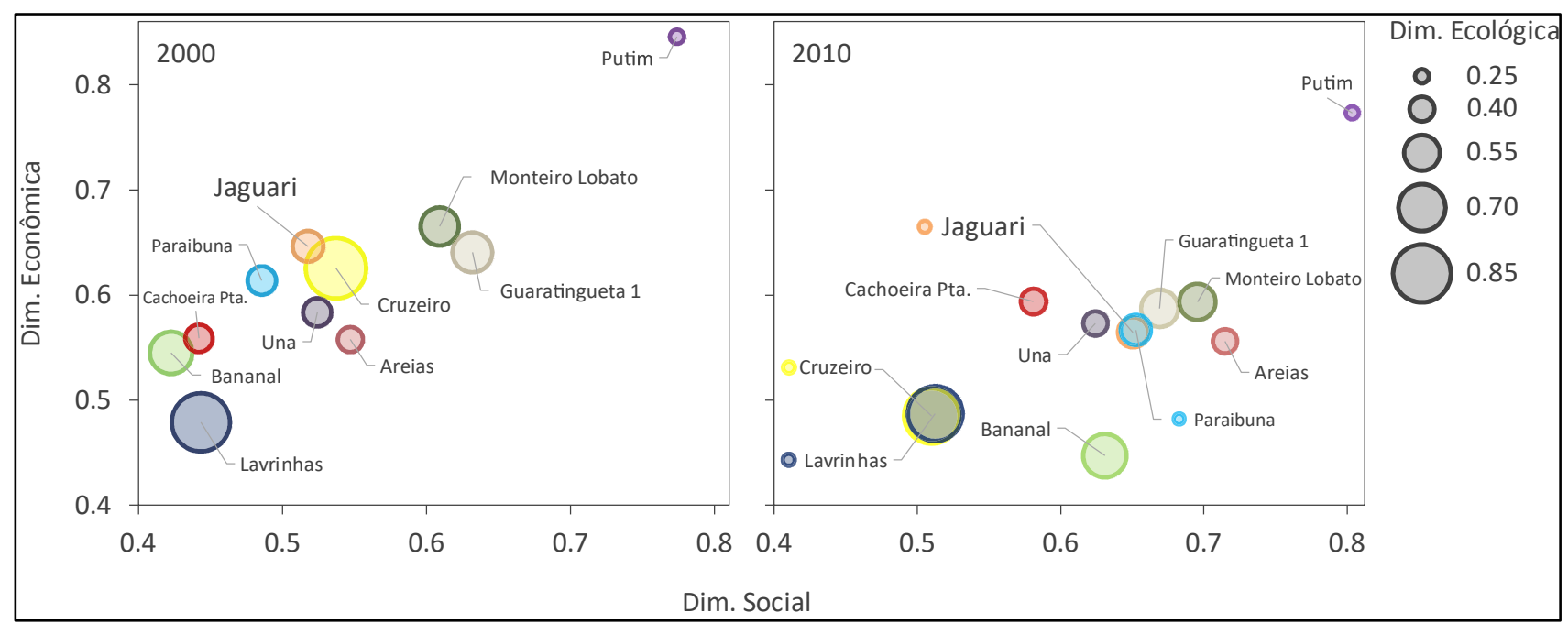

Figura 1: Comparação entre as unidades da APA-MRPS, considerando seus indicadores compostos das dimensões Ecológica (tamanho das bolhas), Social, (eixo $x$ ), e Econômica (eixo y). Com destaque em negrito para a unidade Jaguari, que foi selecionada para análise individual. Fonte: Produzido pelos autores.

\section{Unidade Jaguari}

A Figura 3 apresenta o painel de indicadores da unidade Jaguari, que está parcialmente inserida na Região Metropolitana de São Paulo, a maior metrópole do Brasil, e uma das regiões metropolitanas mais populosas do mundo, com cerca de 20 milhões de habitantes em 2010, dos quais 98,86\% em situação urbana (IBGE, 2010). Esta unidade compreende os núcleos urbanos de Santa Isabel e Igaratá, além de parte dos municípios de Arujá, Guarulhos, Jacareí e São José dos Campos.

$\mathrm{O}$ indicador composto da dimensão Ecológica apresentou uma pequena melhora de 0,47 para 0,49 entre 2000 e 2010. Em 1985 as áreas de 'Pasto/Gramíneas' eram predominantes, mas ao longo do tempo a cobertura diminuiu em extensão, dando lugar a classes em expansão como 'Áreas construídas', 'Florestas' e 'Florestas monoespecíficas'. As 'Áreas construídas' correspondiam 1,2\% da unidade em 1985 e se expandiram em 2015 $(3,3 \%)$. Grande parte das manchas de 'Áreas construídas' localizadas principalmente às margens do reservatório do Jaguari. A área coberta por 'Florestas' decresceu entre 1985 e 1991, mas aumentou continuamente $(9,4 \%)$ nos períodos seguintes, atingindo 31,2\% da área em 2015. As áreas de 'Floresta monoespecífica' também experimentaram crescimento (158,7\%), que ocorreu, principalmente, na década de 2000. Jaguari registrou o maior número de minerações: quatro em Santa Isabel, uma em Arujá e uma em Guarulhos. Destas, apenas uma não existia anterior ao período analisado e todas apresentaram aumento de área (35,7\% de acréscimo). 


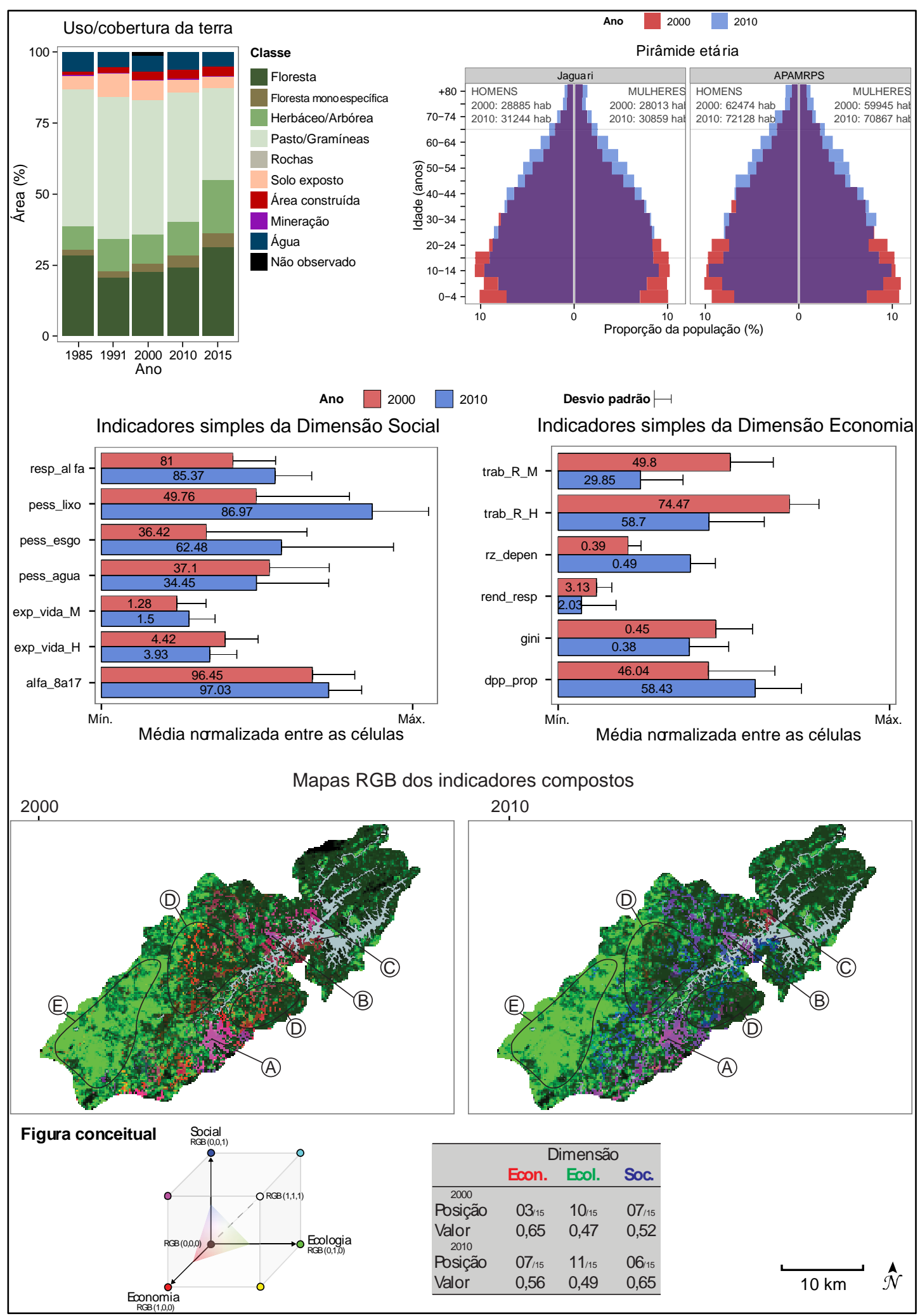

Figura 2: Painel de indicadores da unidade Jaguari. Fonte: Produzido pelos autores.

Na unidade Jaguari, a população residente, predominantemente masculina, aumentou a uma taxa de $0,88 \%$ a.a., de 56.898 habitantes em 2000 para 62.103 em 2010, ou cerca de $43,0 \%$ da população da APA-MRPS. A densidade demográfica de $81,9 \mathrm{hab} / \mathrm{km}^{2}$ passou a $89,4 \mathrm{hab} / \mathrm{km}^{2} \mathrm{em} 2010$.

O indicador sintético da dimensão Social aumentou de 0,52 em 2000 para 0,65 em 2010. A alfabetização dos responsáveis aumentou de $81,0 \%$ para $85,4 \%$, e entre os jovens, de $96,5 \%$ para $97,0 \%$. Observou-se maior proporção de homens que mulheres acima da expectativa de vida, mas esta proporção de mulheres aumentou no período. O saneamento básico apresentou deficiências em abrangência, mas melhorou em dois dos três indicadores entre 2000 e 2010. A cobertura de coleta de lixo cresceu de apenas 49,8\% para 87,0\%. A cobertura 
da coleta de esgotos de apenas 36,4\% melhorou para 62,5\%. O indicador de acesso a água foi o único a reduzir de $37,1 \%$ para $34,5 \%$. Os altos valores de dispersão das métricas das condições de saneamento indicam desigualdade de acesso da população à coleta de lixo e esgoto, e abastecimento de água entre regiões distintas da unidade.

A dimensão Econômica, por sua vez, apresentou queda para o indicador sintético de 0,65 para 0,56. A proporção de mulheres que buscam o mercado de trabalho e são remuneradas com pelo menos um salário mínimo é menor que entre os homens, e a diferença aumentou no período, apontado pela redução dos indicadores de acesso ao mercado de trabalho para as mulheres $(-40,1 \%)$ e homens $(-21,2 \%)$. A razão de dependência cresceu de 0,39 para 0,49 e o rendimento médio do responsável diminuiu no período de 3,1 sm para 2,0 sm. Porém, o número de domicílios próprios aumentou de 46,0\% para 58,4\%. O coeficiente de Gini, que apresentou valores elevados, indicando má distribuição de renda, melhorou de 0,45 para 0,38.

Os mapas em RGB dos indicadores compostos apresentam a distribuição espacial dos diferenciais sociais, econômicos e ecológicos na unidade Jaguari. As regiões mais populosas são os núcleos urbanos de Santa Isabel (A) e Igaratá (B), que em 2000 detinham valores altos para as dimensões Social e Econômica e em 2010 houve decréscimos da dimensão Econômica e crescimento da Social. Às margens do reservatório do Jaguari, há áreas ocupadas (C), que apresentaram valores baixos a intermediários para a dimensão Social, especialmente pela ausência de saneamento básico adequado. Nestas regiões localizam-se condomínios habitacionais de alto padrão, que se desenvolveram em áreas de preservação permanente (APP) às margens do reservatório. A área rural de Santa Isabel (D) apresentou valores altos para a dimensão Econômica e baixos para a Social em 2000, mas em 2010 ambas as dimensões apresentaram valores baixos. Na Serra do Itaberaba (E), observaram-se as melhores condições ecológicas, em função da cobertura predominante ser 'Floresta'. Essa região sobrepõe-se à UC de proteção integral Parque Estadual de Itaberaba, criada em 2010 com o objetivo de conservação da biodiversidade e proteção de espécies ameaçadas do Contínuo Cantareira.

\section{CONSIDERAÇÕES FINAIS}

Este trabalho propôs construir um sistema de indicadores que auxiliasse o diagnóstico socioambiental da APA-MRPS, de modo a fornecer ferramentas para monitorar e avaliar esta UC. O modelo conceitual tomou como base os elementos das definições normativas da APA-MRPS e apresentou uma proposta metodológica para sua operacionalização através da constituição de um sistema de indicadores - o iSAM, e um conjunto de representações espacialmente explícitas que constituem uma cartografia integrada para o monitoramento e planejamento territorial da UC.

Os resultados gerais permitem afirmar que em nenhuma das unidades se observou o equilíbrio desejado entre a preservação da função ecológica relativa aos mananciais e as possibilidades de desenvolvimento social das populações na bacia e seu entorno. Este equilíbrio, na prática, representa a utopia necessária para a construção de um modelo de desenvolvimento socialmente justo e ambientalmente responsável, para o qual os instrumentos propostos neste trabalho, ao iluminar estes desequilíbrios, podem apoiar a constituição de um planejamento ambiental de base territorial que inclua o componente populacional em sua abordagem.

Para avaliação e monitoramento das UCs brasileiras, o iSAM apresenta-se como um método sistemático de monitoramento alternativo àqueles atualmente em uso. A abordagem para concepção do iSAM contempla em sua concepção, a função ecológica de proteção do recurso água e suas características socioeconômicas e demográficas de forma integrada, parte dos processos que regem a dinâmica deste espaço protegido e identifica e localiza no espaço os diferenciais sociais, econômicos e ecológicos, particularizando os diversos contextos existentes. Essa metodologia não tem a pretensão de substituir as iniciativas em curso, ao contrário, configurase como instrumento complementar para apoiar os gestores na ampliação do conhecimento sobre a APAMRPS.

Apesar de sua concepção considerar as particularidades da APA-MRPS, o arcabouço conceitual e metodológico criado, com adaptações, pode ser utilizado para a construção de outros sistemas de indicadores no contexto do monitoramento e planejamento territorial de outras áreas protegidas.

Finalmente, este trabalho descreve as informações e procedimentos metodológicos necessários para a construção e reprodução do iSAM. Todos os dados utilizados são abertos e estão disponíveis para acesso, bem como a maioria das ferramentas computacionais. O esforço a ser feito para a implementação e utilização operacional do iSAM concerne à capacitação dos recursos humanos para sua atualização e adaptações. Esperase ainda que após a criação do plano de manejo da APA-MRPS, o iSAM seja útil para o monitoramento e avaliação da efetividade da gestão. 


\section{REFERÊNCIAS}

AGÊNCIA NACIONAL DE ÁGUAS (ANA). Lista de termos para o Thesaurus de recursos hídricos. Brasília: ANA, 2015.

AGEVAP. Plano integrado de resursos hídricos da bacia hidrográfica do rio Paraíba do Sul e planos de ação de recursos hídricos das bacias afluentes. Resende, RJ: COHIDRO, 2014.

ALLAN, J. D. Landscapes and Riverscapes: The Influence of Land Use on Stream Ecosystems. Annual Review of Ecology, Evolution, and Systematics, v. 35, n. 2004, p. 257-284, 2004.

AMARAL, S.; GAVLAK, A. A.; EESCADA, M. I. S.; MONTEIRO, A. M. V. Using remote sensing and census tract data to improve representation of population spatial distribution: case studies in the Brazilian Amazon. Population and Environment, v. 34, n. 1, p. 142-170, 31 mar. 2012.

ANAZAWA, T. M. Vulnerabilidade e território no litoral norte de São Paulo: indicadores, perfis de ativos e trajetórias. 2012. 190 f. INPE, São José dos Campos, 2012.

BENSUSAN, N. Conservação da biodiversidade em áreas protegidas. Rio de Janeiro: FGV, 2006.

BRASIL. Decreto n. 87.561, de 13 de setembro de 1982. Dispõe sobre as medidas de recuperação e proteção ambiental da Bacia Hidrográfica do Rio Paraíba do Sul, e dá outras providências. Coleção de Leis do Brasil. Brasil: 1982. v. 6. p. 221.

BRASIL. SNUC Sistema Nacional de Unidades de conservação: texto da Lei 9.985 de 18 de julho de 2000 e vetos da presidência da República ao PL aprovado pelo congresso Nacional. 2a ed. São Paulo: Conselho Nacional da Reserva da Biosfera da Mata Atlântica, 2000.

BROCKERHOFF, E. G.; JACTEL, H.; PARROTA, J. A.; QUINE, C. P. SAYER, J. Plantation forests and biodiversity: oxymoron or opportunity? Biodiversity and Conservation, v. 17, n. 5, p. 925-951, 9 maio 2008.

CEEIVAP. Diretrizes de Ordenamento de Uso do Solo: Macro Zoneamento - Síntese do Relatório n. 5. Comitê Especial de Estudos Integrados da Bacia do Rio Paraíba do Sul - CEEIVAP, 1980.

D’ANTONA, A. O.; BUENO, M. C. D.; DAGNINO, R. S. Estimativa da população em unidades de conservação na Amazônia Legal brasileira -- uma aplicação de grades regulares a partir da Contagem 2007. Revista Brasileira de Estatisticas Populacionais, v. 30, n. 2, p. 401-428, 2013.

DRUCK, S.; CÂMARA, G.; MONTEIRO, A. M. V.; CARVALHO, M. S. (Org.). Análise Espacial de Dados Geográficos. Brasília: EMBRAPA, 2004.

HELLER, L. Relação entre saúde e saneamento na perspectiva do desenvolvimento. Ciência \& Saúde Coletiva, v. 3, n. 2, p. 73-84, 1998.

IBGE. Base de informações do Censo Demográfico 2010: Resultados do Universo por setor censitário. Rio de Janeiro: 2010.

IBGE. Indicadores de desenvolvimento sustentável. Rio de Janeiro: Instituto Brasileiro de Geografia e Estatística - IBGE, 2015.

JANNUZZI, P. M. Indicadores sociais no Brasil: Conceitos, Fontes de Dados e Aplicações. 5. ed. Campinas: Editora Alínea, 2012.

JENSEN, J R. Remote Sensing of the Environment: An Earth Resource Perspective. Pearson Prentice Hall, 2007.

LIMA, W. P. Silvicultura e a água: ciência, dogmas, desafios. Rio de Janeiro: Instituto BioAtlântica, 2010.

LIMA, W. P.; FERRAZ, S. F. B.; FERRAZ, K. M. P. M. B. Interações bióticas e abióticas na paisagem: uma perspectiva eco-hidrológica. In: CALIJURI, M. C.; CUNHA, D. G. F. (Org.). Engenharia ambiental: conceitos, tecnologia e gestão. Rio de Janeiro: Elsevier, 2013. p. 215-244.

MARINELLI, C. E. De olho nas unidades de conservação: Sistema de Indicadores Socioambientais para Unidades de Conservação da Amazônia Brasileira. São Paulo: Instituto Socioambiental, 2011.

MARSHALL, R. J. Mapping Disease and Mortality Rates Using Empirical Bayes Estimators. Applied Statistics, v. 40, n. 2, p. 283, 1991. 
NAGENDRA, H.; LUCAS, R.; HONRADO, J. P.; JONGMAN, R. H. G.; TARANTINO, C.; ADAMO, M.; MAIROTA, P. Remote sensing for conservation monitoring: Assessing protected areas, habitat extent, habitat condition, species diversity, and threats. Ecological Indicators, v. 33, p. 45-59, 2013.

PENNINGTON, P.I; LAFFAN, M. Evaluation of the use of pre- and post-harvest bulk density measurements in wet Eucalyptus obliqua forest in Southern Tasmania. Ecological Indicators, v. 4, n. 1, p. 39-54, mar. 2004.

SAATY, T. L. A scaling method for priorities in hierarchical structures. Journal of Mathematical Psychology, v. 15, n. 3, p. 234-281, 1977.

SACHS, I. Desenvolvimento: includente, sustentável, sustentado. Rio de Janeiro: Garamond, 2008.

SIANI, S. M. O. iSAM - Um sistema de indicadores para o monitoramento da área de proteção ambiental mananciais do rio Paraíba do Sul. 2016. 191 p. Dissertação (Mestrado em Sensoriamento Remoto) Instituto Nacional de Pesquisas Espaciais (INPE), São José dos Campos, 2016.

STOTT, T.; LEEKS, G.; MARKS, S.; SAWYER, A. Environmentally sensitive plot-scale timber harvesting: impacts on suspended sediment, bedload and bank erosion dynamics. Journal of environmental management, v. 63, n. 1, p. 3-25, 2001.

TUCCI, C. E. M.; MENDES, C. A. Avaliação ambiental integrada de bacia hidrográfica. Brasília: Ministério do Meio Ambiente - MMA, 2006.

UNITED NATIONS. Indicators of Sustainable Development: Guidelines and Methodologies. Third Edit ed. New York: [s.n.], 2007.

WENDLAND, E; MARIN, I. S. P. Contaminação de águas subterrâneas. In: CALIJURI, M. C.; CUNHA, D. G. F. (Org.). Engenharia ambiental: conceitos, tecnologia e gestão. Rio de Janeiro: Elsevier, 2013. p. 269-293. 